\title{
FGF21 is not required for glucose homeostasis, ketosis or tumour suppression associated with ketogenic diets in mice
}

\author{
Kerstin Stemmer ${ }^{1} \cdot$ Fabio Zani ${ }^{1} \cdot$ Kirk M. Habegger $^{2} \cdot$ Christina Neff $^{1}$ • \\ Petra Kotzbeck $^{1}$ - Michaela Bauer ${ }^{1}$ - Suma Yalamanchilli ${ }^{1}$. Ali Azad ${ }^{3}$ - Maarit Lehti ${ }^{4}$. \\ Paulo J. F. Martins ${ }^{5} \cdot$ Timo D. Müller ${ }^{6} \cdot$ Paul T. Pfluger $^{7} \cdot$ Randy J. Seeley $^{8}$
}

Received: 1 December 2014 / Accepted: 26 May 2015 /Published online: 23 June 2015

(C) Springer-Verlag Berlin Heidelberg 2015

\begin{abstract}
Aims/hypothesis Ketogenic diets (KDs) have increasingly gained attention as effective means for weight loss and potential adjunctive treatment of cancer. The metabolic benefits of KDs are regularly ascribed to enhanced hepatic secretion of fibroblast growth factor 21 (FGF21) and its systemic effects on fatty-acid oxidation, energy expenditure (EE) and body weight. Ambiguous data from Fgf21-knockout animal strains and low FGF21 concentrations reported in humans with ketosis have nevertheless cast doubt regarding the endogenous function of FGF21. We here aimed to elucidate the causal role of FGF21 in mediating the therapeutic benefits of KDs on metabolism and cancer.

Methods We established a dietary model of increased vs decreased FGF21 by feeding C57BL/6J mice with KDs, either depleted of protein or enriched with protein. We furthermore used wild-type and Fgf21-knockout mice that were subjected to the respective diets, and monitored energy and glucose
\end{abstract}

Electronic supplementary material The online version of this article (doi:10.1007/s00125-015-3668-7) contains peer-reviewed but unedited supplementary material, which is available to authorised users.

Randy J. Seeley

seeleyrj@umich.edu

1 Division of Metabolism and Cancer, Institute for Diabetes and Obesity, Helmholtz Centre Munich, Neuherberg, Germany

2 Comprehensive Diabetes Center and Department of MedicineEndocrinology, Diabetes \& Metabolism, University of Alabama at Birmingham, Birmingham, AL, USA

3 Department of Internal Medicine, Division of Endocrinology, Diabetes and Metabolism, University of Cincinnati, Cincinnati, $\mathrm{OH}$, USA homeostasis as well as tumour growth after transplantation of Lewis lung carcinoma cells.

Results Hepatic and circulating, but not adipose tissue, FGF21 levels were profoundly increased by protein starvation, independent of the state of ketosis. We demonstrate that endogenous FGF21 is not essential for the maintenance of normoglycaemia upon protein and carbohydrate starvation and is therefore not needed for the effects of KDs on EE. Furthermore, the tumour-suppressing effects of KDs were independent of FGF21 and, rather, driven by concomitant protein and carbohydrate starvation.

Conclusions/interpretation Our data indicate that the multiple systemic effects of KD exposure in mice, previously ascribed to increased FGF21 secretion, are rather a consequence of protein malnutrition.

Keywords Energy expenditure · Fibroblast growth factor 21 . Glucose homeostasis · Ketogenic diets · Protein starvation . Tumour suppression
4 LIKES Research Center for Sport and Health Sciences, Jyväskylä, Finland

5 Division of Hematology-Oncology, Department of Internal Medicine, Metabolic Diseases Institute, University of Cincinnati, Cincinnati, OH, USA

6 Division of Molecular Pharmacology, Institute for Diabetes and Obesity, Helmholtz Centre Munich, Neuherberg, Germany

7 Research Unit NeuroBiology of Diabetes, Helmholtz Centre Munich, Neuherberg, Germany

8 Department of Surgery, University of Michigan, North Campus Research Center, 2800 Plymouth Road, Ann Arbor, MI 48109-2800, USA 


$\begin{array}{ll}\text { Abbreviations } \\ \text { EE } & \text { Energy expenditure } \\ \text { FGF21 } & \text { Fibroblast growth factor 21 } \\ \text { 3-HB } & \text { 3-Hydroxybutyrate } \\ \text { KD } & \text { Ketogenic diet } \\ \text { KO } & \text { Knockout } \\ \text { LFD } & \text { Low-fat diet } \\ \text { LLC } & \text { Lewis lung carcinoma } \\ \text { LP-KD } & \text { Low-protein KD } \\ \text { MEM } & \text { Minimal essential medium } \\ \text { NEAS } & \text { Non-essential amino acids } \\ \text { PEPCK } & \text { Phosphoenolpyruvate carboxykinase } \\ \text { RP-KD } & \text { Regular-protein KD } \\ \text { WT } & \text { Wild-type }\end{array}$

\section{Introduction}

Ketogenic diets (KDs) are actively used for weight loss [1-4] and anti-epilepsy therapy [5]. More recently KDs have been studied intensively as potential adjunctive treatment for neurodegenerative diseases [6] and brain malignancies [7-9]. The metabolic benefits of KDs have been ascribed to an increased hepatic expression and higher circulating levels of fibroblast growth factor 21 (FGF21) [10-12]. By enhancing fatty-acid oxidation and ketogenesis in response to fasting and KD feeding in rodents, FGF21 has been suggested as a critical component of the metabolic adaptations to a state of fasting and ketosis [10-13]. Profound variations in body weight, fat mass and glucose and lipid metabolism in various strains of Fgf21-knockout (Fgf21-KO) mice [12-16] and the wide range of serum FGF21 concentrations in humans challenged the relevance of endogenous FGF21 in metabolism [17-19]. Nevertheless, the pharmacological application of FGF21 holds promise as an effective therapeutic means for treating obesity and diabetes [20-25] despite uncertainty remaining regarding its physiological role as endocrine mediator of ketogenic adaptations.

We recently showed that KDs need to be low in carbohydrate and protein to trigger weight loss and ketosis in rats [26]. Accordingly, we hypothesised that the metabolic benefits of KDs on energy expenditure (EE), glucose and ketone-body metabolism result from protein starvation rather than directly from increased endogenous FGF21. We further hypothesised that protein starvation, but not FGF21, drives the therapeutic effects of KDs on tumour suppression.

To test these hypotheses, we studied male C57BL/6J mice fed a control low-fat diet (LFD) or KDs containing regular (RP-KD) or low (LP-KD) amounts of protein. We observed that circulating FGF21 levels were elevated only in LP-KDfed mice, while increased ketone-body levels as well as enhanced expression and activity of renal gluconeogenic enzymes were found in both LP-KD- and RP-KD-fed mice. Acute effects of KDs on EE were similar in wild-type (WT) and global Fgf21-KO mice. Furthermore, FGF21 was dispensable for the maintenance of euglycaemia and for ketone-body synthesis upon KD feeding. Finally, we verified that the tumour-suppressing effects of KDs were independent of FGF21 and were mainly determined by carbohydrate and protein starvation.

\section{Methods}

Mice Male 8-week-old C57BL/6J mice (Jackson Laboratory, Bar Harbor, MA, USA) had ad libitum access to LFD (13\% energy from protein, $10 \%$ from fat, $77 \%$ from carbohydrates), an LFD-matched RP-KD (13\% energy from protein, $87 \%$ from fat, $0 \%$ from carbohydrates) or an LFD-matched LPKD content $[10,27,28]$ (5\% energy from protein, $95 \%$ from fat, $0 \%$ from carbohydrates). Diet compositions (Research Diets, New Brunswick, NJ, USA) are shown in electronic supplementary material (ESM) Table 1. Experimental cohort 1 received LFD $(n=9)$, RP-KD $(n=15)$ or LP-KD $(n=15)$ for 8 weeks. Cohort 2 received LFD $(n=10)$, RP-KD $(n=10)$ or LP-KD $(n=10)$ for 2 weeks. Cohorts 3, 4 and 5, consisting of Fgf21-KO mice $[15,29]$ and WT littermates, received LFD $(n=8)$, RP-KD $(n=8)$ or LP-KD $(n=8)$, respectively, for 2 weeks. Before experiments, groups were evenly distributed according to body weight. All experiments were done nonblinded. All procedures for animal use were approved by the University of Cincinnati Institutional Animal Care and Use Committee.

Body composition Body composition was analysed using nuclear magnetic resonance (Echo Medical Systems, Houston, TX, USA).

Oral glucose tolerance test After $6 \mathrm{~h}$ of fasting, mice received an oral bolus of $1.5 \mathrm{~g}$ glucose per $\mathrm{kg}$ lean mass. Blood glucose was measured at 0, 15, 30, 60 and $120 \mathrm{~min}$ by using a glucose analyser (Accucheck; Roche, Indianapolis, IN, USA). Plasma insulin from blood taken at 0 and $15 \mathrm{~min}$ was assessed by ELISA (CrystalChem, Downers Grove, IL, USA).

Pyruvate tolerance test WT and $F g f 21$-KO mice that had been fed for 2 weeks with LFD, RP-KD or LP-KD were fasted for $16 \mathrm{~h}$ and then injected intraperitoneally with $1.5 \mathrm{~g}$ sodium pyruvate (Sigma Aldrich, St Louis, MO, USA) per kg body weight. Tail blood was taken at $0,15,30,60$ and $120 \mathrm{~min}$ and glucose levels were determined with an Accucheck glucose analyser. 
EE The EE, locomotor activity, RQ and food and water intake were measured by combined indirect calorimetry (PhenoMaster; TSE Systems, Bad Homburg, Germany) as described previously [30]. Details are given in ESM Methods.

Tumour cell injection WT and Fgf 21 -KO mice received subcutaneous injections of $4 \times 10^{5}$ cells from the murine Lewis lung cancer cell line (LLC1; ATCC, Manassas, VA, USA), dissolved in PBS. Mice were subsequently switched from chow to LFD $(n=8)$, RP-KD $(n=8)$ or LP-KD $(n=8)$. Tumours were excised and weighed 14 days after tumour cell injection. One LP-KD fed WT mouse died before the end of the experiment and was excluded from the analysis.

Plasma analyses Trunk blood from ad libitum fed mice was collected for plasma preparation. Plasma FGF21 was assessed by FGF21-ELISA (Millipore, Billerica, MA, USA). 3-Hydroxybutyrate (3-HB) was quantified using the colorimetric Autokit 3-HB (Wako, Richmond, VA, USA).

\section{Hepatic and renal phosphoenolpyruvate carboxykinase} activity assay Phosphoenolpyruvate carboxykinase (PEPCK) activity was measured as described previously [31, 32], with modifications detailed in the ESM Methods.

Cell culture experiments HepG2 cells (ATCC HB-8065) were seeded in six-well plates and cultured in minimal essential medium (MEM, M5650; Sigma Aldrich) supplemented with $10 \%$ (vol./vol.) FBS until reaching confluence. Cells were subsequently washed with PBS and incubated for $6 \mathrm{~h}$ in serum-free MEM with or without non-essential amino acids (M5650 and M2279; Sigma Aldrich).

Gene expression analyses Quantitative gene expression analyses from 7-14 random samples per group were performed as described $[33,34]$ using gene-specific TaqMan probes (ESM Table 2). The relative expression of each gene was normalised to that of Actb or Pgkl [34].

Statistics Data are reported as means \pm SEM. Statistical differences were assessed by one-way or two-way ANOVA with Tukey's post hoc tests. EE was analysed by ANCOVA using fat and lean mass as covariates and Bonferroni post hoc tests. A $p$ value $\leq 0.05$ was regarded as statistically significant. All analyses were performed using Prism 6.0 (GraphPad, La Jolla, CA, USA) or SPSS 20 (IBM, Armonk, NY, USA).

\section{Results}

Body weight changes in response to KDs depend on the relative amounts of dietary fat and protein WT C57BL/6 mice were ad libitum fed with LFD, RP-KD or LP-KD for 8 weeks. While RP-KD feeding led to a significant increase in body weight compared with LFD-fed mice, prolonged feeding with LP-KD resulted in a significant decrease in body weight (Fig. 1a). The increased body weight in RP-KD-fed mice is in accordance with the significantly increased fat mass after 8 weeks of feeding, while LP-KD-fed mice showed a trend ( $p=0.0525$ ) towards lower body fat content compared with LFD-fed mice (Fig. 1b). Significant lean mass loss after 4 and 8 weeks of LP-KD feeding accompanied the pronounced body weight loss of this diet group (Fig. 1c). Mice consumed smaller amounts of the more energy-dense KDs (Fig. 1d), but the cumulative energy intake was similar in all diet groups (Fig. 1e). Body weight loss in the LP-KD-fed mice correlated with the significant reduction in food efficiency (Fig. 1f) of this diet. Glucose excursion and glucose AUC were significantly lower in LP-KD-fed mice (Fig. 1g, h), as expected based on their significantly lower body weight and body fat. Insulin levels tended to be lower in LP-KD-fed mice (Fig. 1i) than in those fed with an LFD or RP-KD.

Exposure to protein-starved KD results in muscle wasting in mice In accordance with the observed lean mass loss, LP$\mathrm{KD}$ feeding resulted in a significant loss of gastrocnemius wet tissue mass after 1 week (Fig. 2a) and 2 weeks (Fig. 2b) of LPKD feeding. Similarly, soleus wet tissue mass was significantly decreased after 1 week (Fig. 2c) and 2 weeks (Fig. 2d) of LP-KD feeding. Expression of the muscle atrophy marker, muscle RING-finger protein-1 gene Murf1 [35], tended to be increased in the gastrocnemius (Fig. 2e) and soleus (Fig. 2f) muscles of mice fed with LP-KD for 2 weeks, when compared with those fed either RP-KD or LFD. The expression of another muscle atrophy marker, atrogin-1 gene (Fbxo32) [35], was significantly increased in the gastrocnemius (Fig. 2g) and soleus (Fig. 2f) muscles after 2 weeks of LP-KD exposure.

\section{Liver but not adipose tissue FGF21 expression upon ketosis} depends on the amount of dietary protein Plasma levels of 3-HB were increased by 6.9 -fold and 3.8-fold after 8 weeks of feeding mice with LP-KD and RP-KD, respectively, when compared with levels in mice fed LFD (Fig. 3a), indicating that both diets cause a state of ketosis. Expression of mitochondrial Bdhl (the gene encoding D- $\beta$-hydroxybutyrate dehydrogenase, which is involved in the conversion of acetoacetate to 3-HB) was significantly increased in LP-KDfed mice compared with LFD-fed controls (Fig. 3b). RP-KDand LP-KD-fed mice displayed a significant reduction in the expression of $B d h 2$, which is involved in the reversion of 3HB to acetoacetate (Fig. 3c). Previous reports suggest an essential role for FGF21 in hepatic ketogenesis during fasting [12]. Accordingly, our finding of elevated ketone-body secretion in LP-KD-fed mice should be accompanied by elevated FGF21 expression and secretion. However, only the exposure 
a

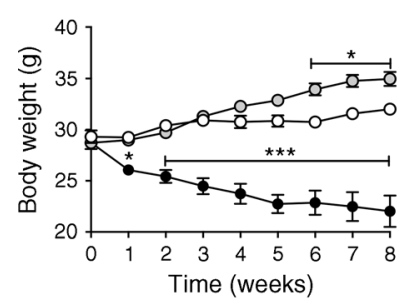

d

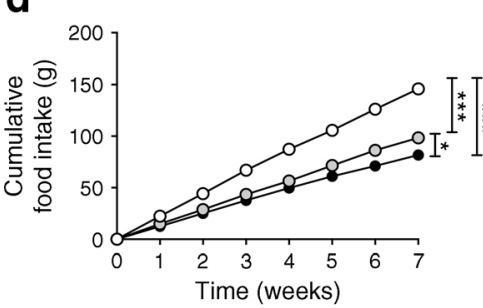

b

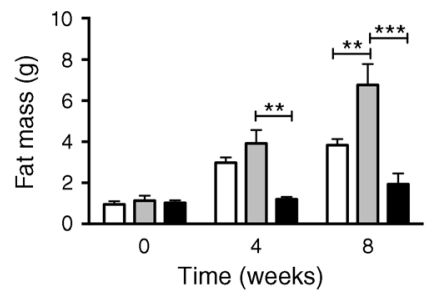

e
C

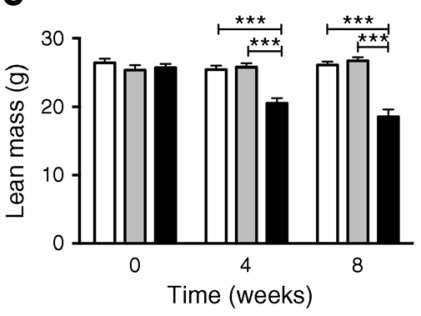

f g

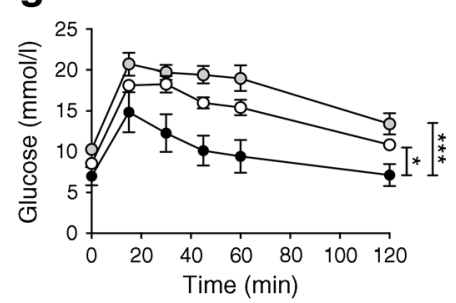

h

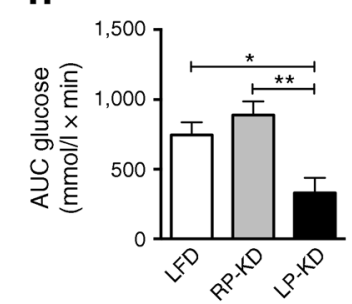

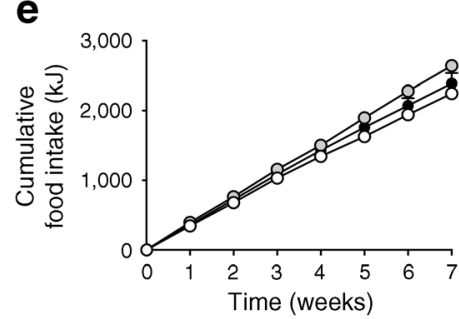

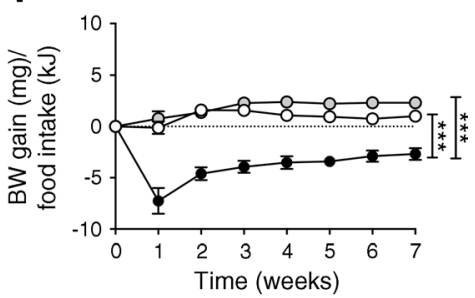

i

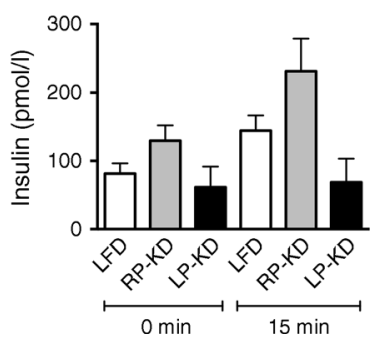

Fig. 1 (a-c) C57BL/6 mice fed with RP-KD for 8 weeks demonstrated increased body weight (a) and fat mass (b) compared with LFD-fed controls. LP-KD-fed mice revealed decreased body weight (a) and lean mass (c). (d, e) Mice consumed smaller amounts of the more energydense KDs (d), resulting in similar energy consumption in all groups (e). (f) Decreased food efficiency was observed in LP-KD-fed mice. BW, body weight. (g-i) LP-KD feeding for 7 weeks decreased glucose

to LP-KD for 8 weeks, and not to RP-KD, resulted in a significant upregulation of liver $F g f 21$ gene expression (Fig. $3 \mathrm{~d}$ ). Differences in the expression of Fgf21 in the liver were reflected by similar differences in plasma FGF21 levels

excursions (g) and blood glucose AUC (h) and lowered insulin levels in an oral glucose tolerance test (i). White circles and bars, LFD; grey circles and bars, RP-KD; black circles and bars, LP-KD. Data are means \pm SEM; $n=9$ (LFD) or $n=15$ (RP-KD and LP-KD) (a-f); $n=7$ random mice per group (g-i). In (a) $* p<0.05, * * * p<0.001$ vs LFD and in (b-i), ${ }^{*} p<0.05$, $* * p<0.01$ and $* * * p<0.001$ for the indicated comparisons

(Fig. 3e). A similar increase in 3-HB levels in Fgf21-KO and WT mice fed with KDs for 2 weeks further confirmed that FGF21 is not essential for ketogenesis (Fig. 3f). To assess whether protein starvation can cause the increase in hepatic

Fig. 2 (a-d) Decreased wet tissue weight of gastrocnemius $(\mathrm{GC})(\mathbf{a}, \mathbf{b})$ and soleus $(\mathbf{c}, \mathbf{d})$ muscle in mice fed LP-KD for 7 $(\mathbf{a}, \mathbf{c})$ and 14 days $(\mathbf{b}, \mathbf{d}) .(\mathbf{e}, \mathbf{f})$ Murf1 expression in the GC (e) and soleus (f) muscle tended to increase after 14 days of LP-KD exposure. (g, h) Fbxo32 was significantly increased in GC (g) and soleus (h) muscle of LP-KDfed mice after 14 days. Data are means \pm SEM, $n=10 .{ }^{*} p<0.05$ and ${ }^{* *} p<0.01$ for the indicated comparisons
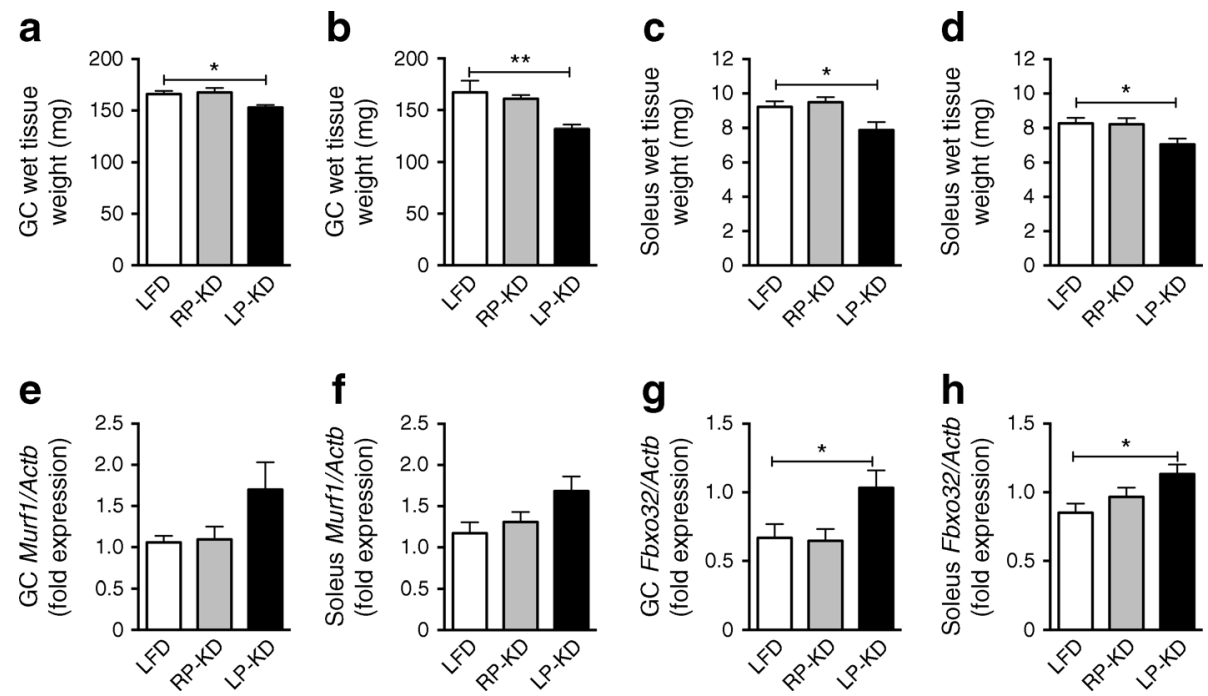

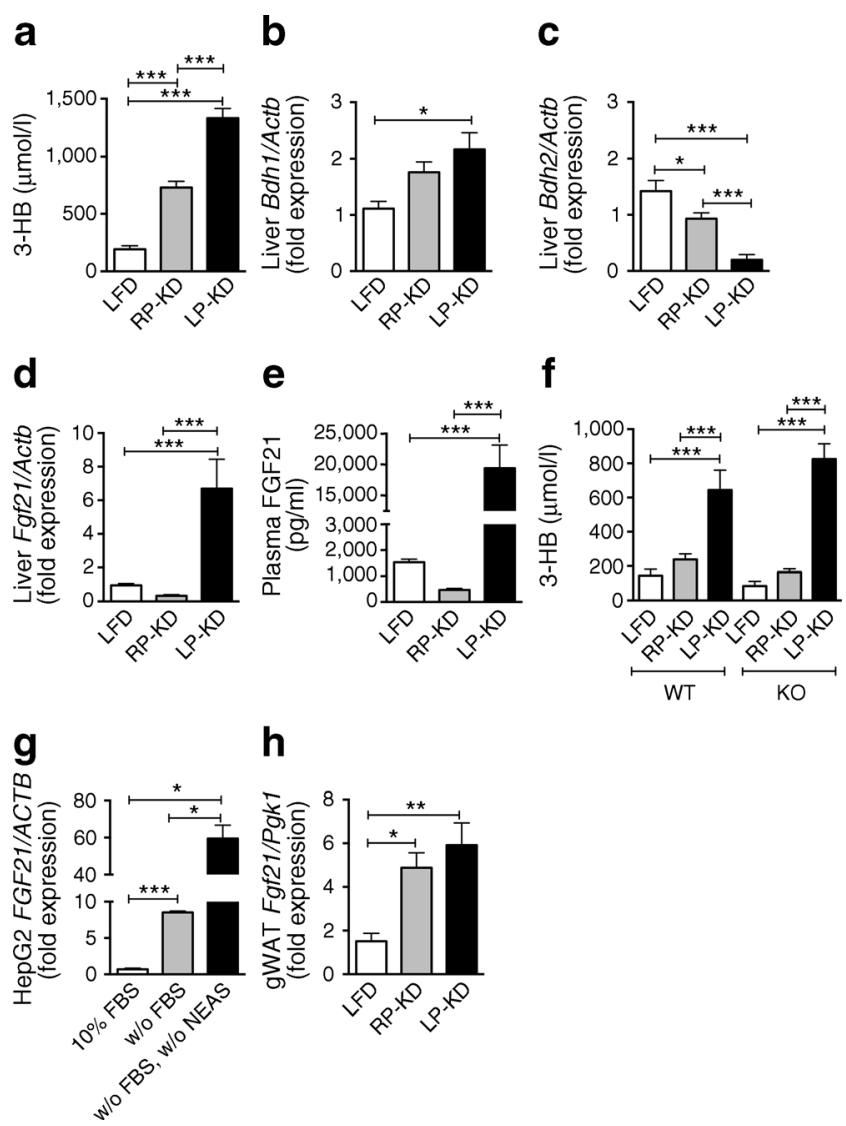

Fig. 3 (a) Increased plasma 3-HB levels in mice fed RP-KD or LP-KD for 8 weeks. (b) Increased hepatic Bdh1 expression in RP-KD-fed mice compared with LFD-fed controls. (c) Reduced hepatic $B d h 2$ expression in RP-KD- and LP-KD-fed mice compared with LFD-fed controls. (d, e) Higher hepatic Fgf21 expression (d) and plasma FGF21 levels (e) in LPKD-fed mice compared with LFD- and RP-KD-fed mice. (f) Similar and significant increase in plasma levels of 3-HB in WT and Fgf21-KO mice following 2 weeks of LP-KD feeding. (g) Increased $F G F 21$ expression in HepG2 cells following concomitant FBS and NEAS starvation (w/o FBS, w/o NEAS), compared with FBS-starved cells (w/o FBS) and control cells $(10 \%$ FBS). (h) Significant increase in Fgf 21 expression in the gonadal white adipose tissue (gWAT) of RP-KFD- and LP-KD-fed mice. Data are means \pm SEM, $n=7-14$. For cell culture experiments, three independent wells derived from three different six-well plates were analysed. $* p<0.05, * * p<0.01$ and $* * * p<0.001$ for the indicated comparisons

FGF21 expression, HepG2 liver cells were starved selectively of non-essential amino acids (NEAS) or non-selectively by deprivation of FBS. The lack of FBS caused a slight but significant increase in FGF21 expression (Fig. 3g). Additional reduction in NEAS resulted in a pronounced upregulation of $F G F 21$ expression compared with its expression under conditions of FBS starvation (Fig. 3g). Fgf21 expression was significantly increased in gonadal white adipose tissue of both RP-KD- and LP-KD-fed mice (Fig. 3h) and thus was independent of the protein content in the KD.

Short-term dietary effects of KDs on EE are independent of endogenous FGF21 Weight-matched WT (Fig. 4a) and
Fgf21-KO mice (Fig. 4b) had a similar RQ when fed regular chow diet and the RQ decreased when these mice were acutely exposed to RP-KD or LP-KD. Chronic exposure of WT mice to LFD or KD for 7 weeks resulted in a similar drop in RQ (Fig. 4c) and corroborated the switch in nutrient partitioning from carbohydrate to lipid oxidation. WT (Fig. 4d) and Fgf21-KO mice (Fig. 4e) displayed similar locomotor activity on chow diet, and reduced activity when exposed to RP-KD or LP-KD for 3 days. Exposure of WT mice to KDs for 7 weeks resulted in a similar decrease in activity when compared with LFD-fed WT controls (Fig. 4f). Further dissection of locomotor patterns revealed that the dark and light phase activity of WT and Fgf21-KO mice on chow diet were similar (Fig. 4g), but there was a drop in dark phase locomotion after acute $\mathrm{KD}$ exposure in WT (Fig. 4h) and Fgf21-KO mice (Fig. 4i), and after 7 weeks of KD exposure in WT mice (Fig. 4j).

EE was similar in animals fed chow-diet (ESM Table 3). Switching the diet to LFD, RP-KD or LP-KD revealed a significant effect on dark phase EE in WT (Fig. 4k) and Fgf21-KO (Fig. 4l) mice after ANCOVA with lean and fat mass as covariates (ESM Fig. 1). The covariate lean mass, but not fat mass, was significantly related to EE (ESM Table 4). Bonferroni post hoc tests showed a significant decrease in dark phase EE in WT mice fed with LP-KD compared with LFD ( $p=0.031)$, and a trend for decreased total EE in WT mice fed with LP-KD compared with mice fed with LFD ( $p=0.053$ ). Similarly, Fgf21-KO mice fed with LP-KD displayed significantly decreased dark phase $\mathrm{EE}(p=0.008)$ when compared with LFD-fed mice. We did not observe any significant interaction effects between genotypes and dietary exposure (Genotype $\times$ Diet for EE total: $F=0.004, p=0.996$; EE dark phase: $F=0.113, p=0.894$; EE light phase: $F=0.078$, $p=0.925$ ), demonstrating that WT and $F g f 21$-KO mice respond similarly to the exposure to LFD, RP-KD or LP-KD, respectively. Interestingly, after 7 weeks of feeding the dietary impact on EE was lost (Fig. 4m); EE continued to be strongly related to lean mass, but LP-KD or RP-KD exposure no longer had a significant effect (ESM Table 4).

FGF21 is not required for maintaining glucose levels upon ketosis Both RP-KD and LP-KD-fed mice were able to maintain normoglycaemia despite the absence of carbohydrates (Fig. 1g). Based on this finding and our findings of muscle catabolism and higher liver Fgf21 expression in LP-KD-fed mice but not RP-KD-fed mice, we hypothesised that FGF21 could be an important factor for muscle protein catabolism to ultimately provide amino acids as substrates for gluconeogenesis.

Following 8 weeks of feeding with LFD or KDs, hepatic expression of genes encoding the gluconeogenic enzymes glucose-6-phosphatase catalytic subunit (G6pc, Fig. 5a) and PEPCK (Pck1, Fig. 5b) was at similar levels under all dietary conditions, as was PEPCK activity (Fig. 5c). Similarly, liver 
a

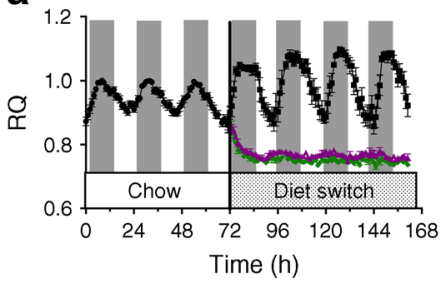

b

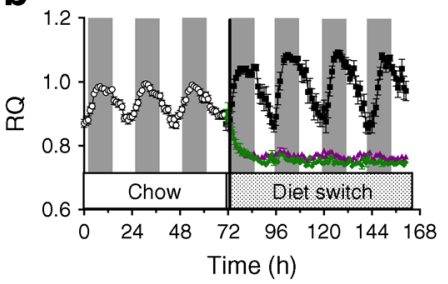

C

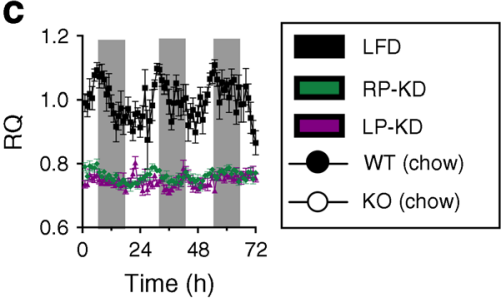

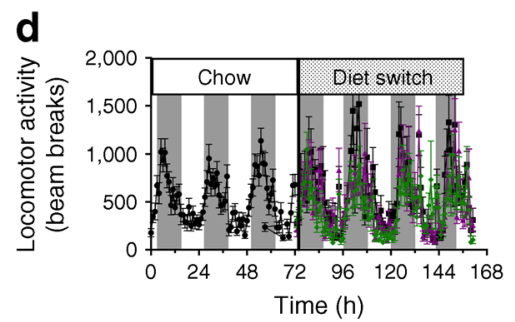
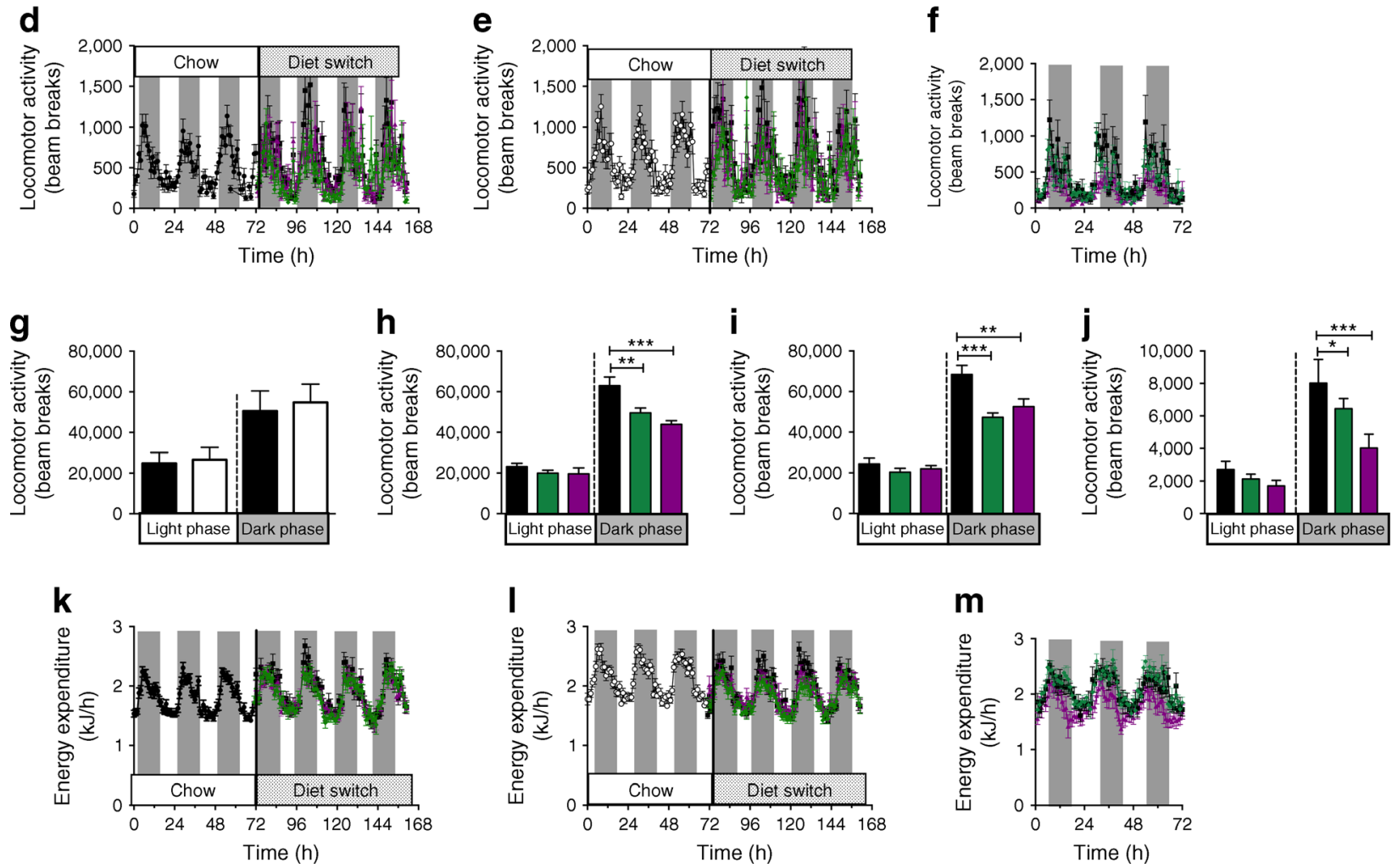

Fig. 4 (a-c) RQ in WT (a) and Fgf2l-KO (b) mice following an acute dietary switch from chow to either LFD, RP-KD or LP-KD, or in WT mice after 7 weeks of LFD, RP-KD or LP-KD exposure (c). (d-f) Locomotor activity in WT (d) and $F g f 21-\mathrm{KO}$ (e) mice exposed to chow, and after an acute switch to LFD, RP-KD or LP-KD. In WT mice, locomotor activity was further monitored after 7 weeks of dietary exposure (f). (g-i) Daily locomotor activity during the dark and light phase in chow-fed WT

(black bars) and $F g f 21-\mathrm{KO}$ mice (white bars) (g) and after the dietary switch in WT and Fgf2l-KO (i) mice. (j) Daily dark phase locomotor activity of WT mice following 7 weeks of LFD, RP-KD or LP-KD exposure. (k-m) Changes in EE in WT (k) and $F g f 21$-KO (l) mice following the dietary switch from chow to LFD, RP-KD or LP-KD, and in WT mice after 7 weeks of LFD, RP-KD or LP-KD (m). Data are means \pm SEM, $n=7-9$. ${ }^{*} p<0.05,{ }^{* *} p<0.01$ and ${ }^{* * *} p<0.001$ for the indicated comparisons

expression of the gene encoding peroxisome proliferatoractivated receptor- $\gamma$ co-activator $\alpha(\operatorname{Pgcl} \alpha$, also known as Ppargcla), a transcriptional co-activator that controls the expression of gluconeogenic genes [36], remained unaffected by diet (ESM Fig. 2). In contrast, 8 weeks of LP-KD feeding resulted in a significant increase in the expression of $G 6 p c$ (Fig. 5d) and Pckl (Fig. 5e) in the kidney. A similar increase in the expression of $P c k 1$, but not $G 6 p c$, in the kidney was also detected in RP-KD-fed mice (Fig. $5 \mathrm{~d}$, e). The increased renal Pckl expression was in accordance with the significant increase in PEPCK activity found in the kidney of RP-KD-fed mice and a trend $(p=0.189)$ towards an increased PEPCK activity in LP-KD-fed mice (Fig. 5f). Notably, expression of the genes encoding fibroblast growth factor receptor $1(F g f r l)$ and $\beta$-klotho $(K l b)$ was found to be increased in renal cortices isolated from LP-KD-fed mice (ESM Fig. 3). Neither substantial differences in hepatic Fgf21 expression (ESM Fig. 4) nor genetic ablation of $F g f 21$ seemed to affect gluconeogenesis, as revealed by similar glucose excursions in pyruvate tolerance tests in WT (Fig. 5g) and Fgf 21 -KO (Fig. 5h) mice and similar ad libitum blood glucose levels (Fig. 5i) in WT and Fgf21-KO mice after 2 weeks of LFD, RP-KD or LP-KD feeding.

Exposure to protein-starved KD suppresses tumour growth independently of FGF21 Previous data indicate that KDs can slow down tumour growth [7, 8]. Using a subcutaneous LLC1 allograft cancer model we demonstrated that the beneficial effect of KDs on tumour growth depends on the diet 

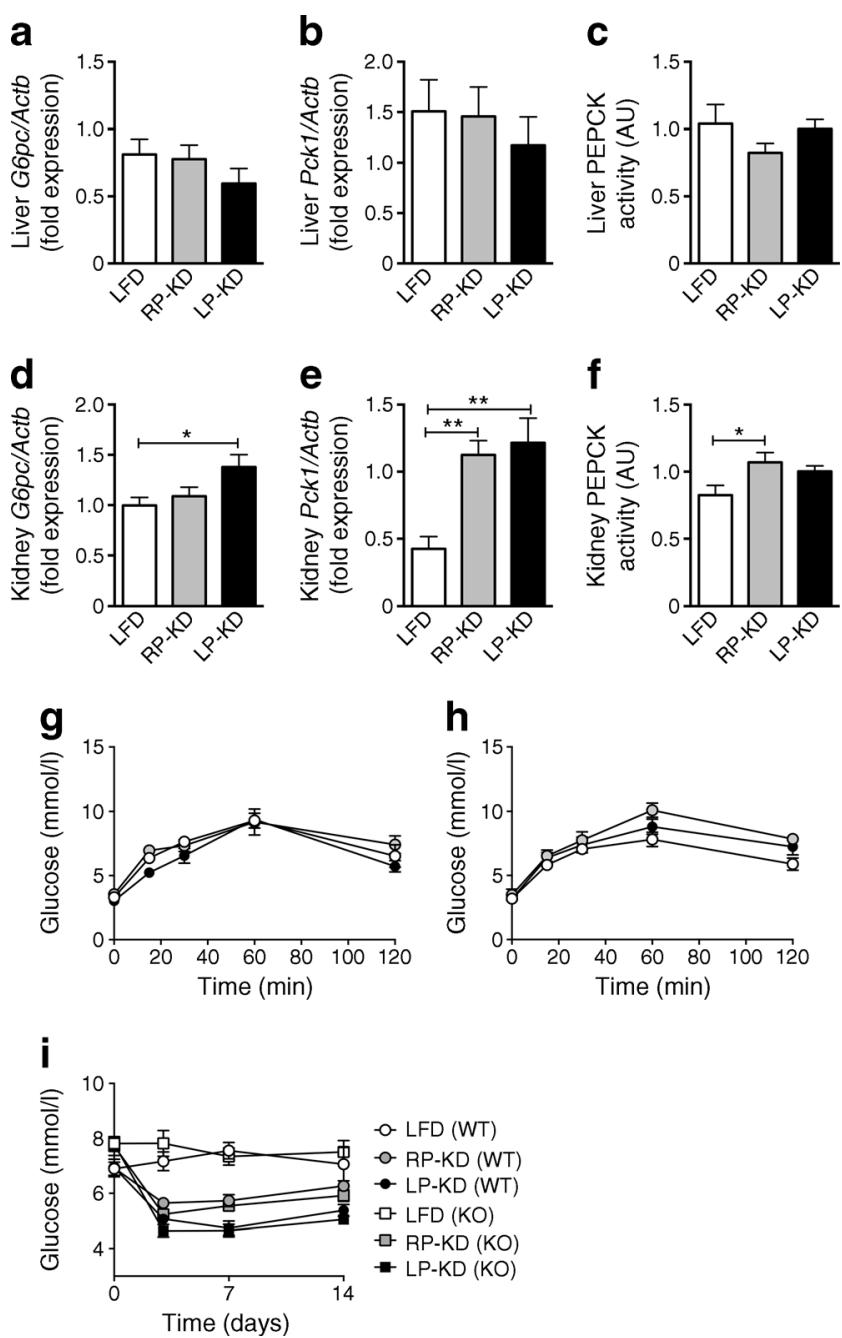

Fig. 5 (a-c) Hepatic expression of G6pc (a) and Pckl (b) and hepatic PEPCK activity (c) in mice after 8 weeks of exposure to LFD, RP-KD and LP-KD. (d-f) Renal cortex expression of G6pc (d) and Pckl (e) and renal PEPCK activity (f). (g, h) Pyruvate tolerance tests in WT (g) and Fgf21-KO mice (h) fed with LFD (white circles), RP-KD (grey circles) or LP-KD (black circles) for 2 weeks. (i) Ad libitum blood glucose of WT (circles) and Fgf21-KO (squares) mice before and after 7 and 14 days of exposure to LFD (white symbols), RP-KD (grey symbols) or LP-KD (black symbols). Data are means \pm SEM, $n=8-10 .{ }^{*} p<0.05$ and $* * p<0.01$ for the indicated comparisons

composition. While the RP-KD did not significantly affect tumour weight, LP-KD feeding for 14 days resulted in a significant reduction in tumour weight in WT mice (Fig. 6). The effect was independent of FGF21 signalling, since similar results were obtained in WT and $F g f 21$-KO mice.

\section{Discussion}

KDs have garnered attention as an effective treatment for obesity by causing weight loss and improving glucose tolerance and plasma lipid profiles [1-4], potentially via release of FGF21 $[10,12]$. Through a series of experiments, we reveal

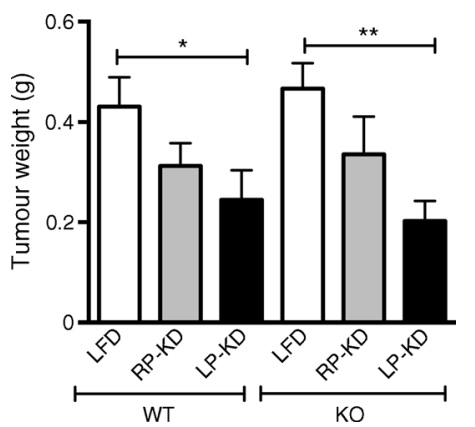

Fig. 6 Tumour weights of subcutaneous LLC1 allografts were reduced by exposure to LP-KD for 14 days in both WT mice and $F g f 21$-KO mice. Data are means \pm SEM, $n=7-8 .{ }^{*} p<0.05$ and ${ }^{*} p p<0.01$ for the indicated comparisons

that FGF21 is not an essential mediator of the metabolic alterations seen with $\mathrm{KD}$ feeding in mice. Our data suggest that the overall protein content in a $\mathrm{KD}$ is an important contributor to its effects on body weight, energy and glucose homeostasis.

We reveal that body weight and lean mass are potently decreased by LP-KD feeding. In contrast, a gain in fat mass in RP-KD-fed mice led to significantly higher body weights compared with LFD- and LP-KD-fed mice. Both LP-KD and RP-KD feeding induced ketosis, as revealed by increased 3-HB levels in RP-KD- and LP-KD-fed mice, compared with LFD controls. However, circulating FGF21 levels were only increased in LP-KD-fed mice compared with RP-KD- or LFD-fed mice. Accordingly, our data corroborate previous findings that hepatic and plasma FGF21 levels are indicative of protein starvation [26, 37-39] but not the state of ketosis [15]. In contrast to the protein-dependent regulation of hepatic Fgf21 expression and FGF21 release, RP-KD- and LP-KDfed mice displayed a similar upregulation of $F g f 21$ in the gonadal white adipose tissue, suggesting alternative regulation of FGF21 in adipose tissue compared with liver.

The mechanisms underlying the weight-lowering effects of KDs remain a matter of debate. In our model, we observed a similar energy intake in all dietary groups but severely diminished food efficiency in LP-KD-fed mice. The inability of LP$\mathrm{KD}$-fed mice to translate ingested energy into weight gain may either be due to malabsorption, urinary loss of ketones or increased EE. Indeed, recent evidence suggests that $\mathrm{KD}$ feeding increases EE $[27,28]$. FGF21 may play a role in this thermogenic effect of KDs, as also suggested by increased EE in obese rats or mice after central or intraperitoneal infusion, respectively, of pharmacological doses of FGF21 [40, 41]. Further, hypothalamic $\beta$-klotho, the obligate co-receptor for the FGF21 receptor fibroblast growth factor receptor 1, is required for the central effects of FGF21 on EE in transgenic Fgf21 mice [42].

To investigate the impact of endogenous FGF21 on EE, we performed indirect calorimetry measurements in WT and Fgf21-KO mice following an acute challenge with LFD, RP- 
KD or LP-KD. The acute feeding paradigm assured that lean mass as predictive covariate for EE was similar for all groups. LP-KD- but not RP-KD-fed mice showed a significant decrease in EE during the dark phase compared with LFD-fed mice. This stands in contrast to previous findings by Leager et al [39] showing increased EE in mice fed a protein-restricted regular diet. Further, in contrast to Laeger et al, who showed that dietary effects on EE were no longer present in an alternative strain of Fgf21-KO mice, we observed a similar decrease in EE in LP-KD-fed KO and WT mice, suggesting that this effect was FGF21-independent [39]. To assess whether these differences were due to a shorter exposure of our mice, we compared C57BL/6 WT mice that were fed chronically with RP-KD and LP-KD and displayed low and high circulating FGF21 levels, respectively. ANCOVA analyses revealed that effects on EE were strongly related to changes in lean mass, but not to LP-KD or RP-KD exposure. Together, our findings do not support a functional role for endogenous FGF21 in EE, at least not in a model of KD exposure.

Current studies investigating the effect of FGF21 on carbohydrate metabolism are controversial. FGF21 has been implicated in the regulation of gluconeogenesis during the progression from fasting to starvation [13]. Administration of recombinant FGF21 induced hepatic expression of key regulators of gluconeogenesis such as Pgcl $\alpha$ and Pck1 but not G6pc [43]. In contrast, therapeutic administration of FGF21 had a potent glucose-lowering effect in $o b / o b$ and $d b / d b$ mice [44]. Glucose output was suppressed in H4IIE hepatoma cells exposed to FGF21 [45].

In our experimental set-up neither the expression of hepatic gluconeogenic enzymes G6pc, Pckl and Pgcl $\alpha$ nor PEPCK activity were altered by 8 weeks of KD feeding. This is consistent with findings from rats fed with KD for 4 weeks [26] and WT and Fgf21-KO mice fed for 6 days with KD [46]. In contrast, G6pc and Pck1 expression, as well as PEPCK activity, was elevated in the kidney cortex of our KD-fed mice. Overall, our data suggest that enhanced renal gluconeogenesis is an important contributor to systemic glucose control in a state of ketosis. However, similar changes in blood glucose levels during 14 days of KD feeding and similar glucose excursions following an acute pyruvate challenge in KDfed WT and Fgf21-KO mice do not support an essential role for endogenous FGF21 in the overall maintenance of normoglycaemia during KD feeding.

We finally aimed to investigate whether FGF21 might be involved in the anti-tumorigenic effects of KD feeding, and implanted LLC1 cells into WT and Fgf21-KO mice fed with LP-KD, RP-KD and LFD. We observed significantly diminished tumour growth in both WT and KO mice after LP-KD feeding, suggesting that concomitant dietary protein and carbohydrate deprivation but not FGF21 are necessary to reduce tumour growth. This finding may have important clinical implications for cancer patients that are subjected to adjuvant KD therapy. The prospect of anti-tumorigenic efficacy being increased by adjuvant LP-KD may be promising for cancer patients and warrants future clinical studies, but adverse clinical symptoms of protein malnutrition should be monitored closely.

In conclusion, our studies demonstrate that endogenous FGF21 is a biomarker for protein starvation but is dispensable for the maintenance of normoglycaemia, ketone-body synthesis and tumour suppression in mice fed a KD. The metabolic alterations in EE and glucose and ketone-body metabolism caused by KD in mice were rather a consequence of concomitant carbohydrate and protein starvation. Future studies will need to address remaining questions such as the correct protein content and composition of KD to induce similar states of ketosis in mice and humans, or the often-discrepant findings obtained by endogenous FGF21 induction vs pharmacological doses of FGF21. Nevertheless, we believe that our physiological model of increased or decreased FGF21 in response to protein-starved or regular-protein $\mathrm{KD}$ provides a useful tool with which to investigate the impact of endogenous FGF21 on the therapeutic effects of KD on metabolism and cancer.

Acknowledgements The authors thank N. Itho (Kyoto University Graduate School of Pharmaceutical Sciences, Sakyo, Kyoto, Japan) for providing the $\mathrm{Fg} 2 \mathrm{2} 1 \mathrm{-KO}$ mice.

Funding This work was supported in part by NIH grant U01 CA141464-02. KS was supported by a fellowship from the German Research Foundation (STE 1466/4-1). KMH was supported by ADA grant 1-13-JF-21.

Duality of interest RJS has received research support from Novo Nordisk, Ethicon Surgical Care, Eisai and Boehringer Ingelheim and has served as a paid consultant for Novo Nordisk, Ethicon Surgical Care, Boehringer Ingelheim, Sanofi, Novartis and Takeda. RJS has equity in Zafgen and Endobetix. All other authors declare that there is no duality of interest associated with their contribution to this manuscript.

Contribution statement $\mathrm{KS}, \mathrm{FZ}, \mathrm{KMH}, \mathrm{CN}, \mathrm{PK}, \mathrm{MB}, \mathrm{SY}, \mathrm{AA}, \mathrm{ML}$, PJFM, TDM, PTP and RJS made substantial contributions to conception and design, acquisition of data, or analysis and interpretation of data. KS and PTP drafted the manuscript. FZ, KMH CN, PK, MB, SY, AA, ML, PJFM, TDM, and RJS critically revised the article for intellectual content. All authors approved the final manuscript. KS is responsible for the integrity of the work as whole.

\section{References}

1. Brehm BJ, Seeley RJ, Daniels SR, D’Alessio DA (2003) A randomized trial comparing a very low carbohydrate diet and a calorierestricted low fat diet on body weight and cardiovascular risk factors in healthy women. J Clin Endocrinol Metab 88:1617-1623

2. Foster GD, Wyatt HR, Hill JO et al (2003) A randomized trial of a low-carbohydrate diet for obesity. N Engl J Med 348:2082-2090 
3. Dashti HM, Mathew TC, Khadada M et al (2007) Beneficial effects of ketogenic diet in obese diabetic subjects. Mol Cell Biochem 302: 249-256

4. Acheson KJ (2010) Carbohydrate for weight and metabolic control: where do we stand? Nutrition 26:141-145

5. Kessler SK, Neal EG, Camfield CS, Kossoff EH (2011) Dietary therapies for epilepsy: future research. Epilepsy Behav 22:17-22

6. Paoli A, Bianco A, Damiani E, Bosco G (2014) Ketogenic diet in neuromuscular and neurodegenerative diseases. Biomed Res Int 2014:474296

7. Nebeling LC, Miraldi F, Shurin SB, Lerner E (1995) Effects of a ketogenic diet on tumor metabolism and nutritional status in pediatric oncology patients: two case reports. J Am Coll Nutr 14:202-208

8. Seyfried TN, Sanderson TM, El-Abbadi MM, McGowan R, Mukherjee P (2003) Role of glucose and ketone bodies in the metabolic control of experimental brain cancer. Br J Cancer 89: $1375-1382$

9. Seyfried TN, Kiebish MA, Marsh J, Shelton LM, Huysentruyt LC, Mukherjee P (1807) Metabolic management of brain cancer. Biochim Biophys Acta 2011:577-594

10. Badman MK, Pissios P, Kennedy AR, Koukos G, Flier JS, MaratosFlier E (2007) Hepatic fibroblast growth factor 21 is regulated by PPARalpha and is a key mediator of hepatic lipid metabolism in ketotic states. Cell Metab 5:426-437

11. Inagaki T, Dutchak $P$, Zhao G et al (2007) Endocrine regulation of the fasting response by PPARalpha-mediated induction of fibroblast growth factor 21. Cell Metab 5:415-425

12. Badman MK, Koester A, Flier JS, Kharitonenkov A, Maratos-Flier E (2009) Fibroblast growth factor 21-deficient mice demonstrate impaired adaptation to ketosis. Endocrinology 150:4931-4940

13. Potthoff MJ, Inagaki T, Satapati S et al (2009) FGF21 induces PGC1alpha and regulates carbohydrate and fatty acid metabolism during the adaptive starvation response. Proc Natl Acad Sci U S A 106: 10853-10858

14. Dutchak PA, Katafuchi T, Bookout AL et al (2012) Fibroblast growth factor-21 regulates PPARgamma activity and the antidiabetic actions of thiazolidinediones. Cell 148:556-567

15. Hotta Y, Nakamura H, Konishi M et al (2009) Fibroblast growth factor 21 regulates lipolysis in white adipose tissue but is not required for ketogenesis and triglyceride clearance in liver. Endocrinology 150:4625-4633

16. Adams AC, Coskun T, Cheng CC, O'Farrell LS, Dubois SL, Kharitonenkov A (2013) Fibroblast growth factor 21 is not required for the antidiabetic actions of the thiazoladinediones. Mol Metab 2: 205-214

17. Galman C, Lundasen T, Kharitonenkov A et al (2008) The circulating metabolic regulator FGF21 is induced by prolonged fasting and PPARalpha activation in man. Cell Metab 8:169-174

18. Christodoulides C, Dyson P, Sprecher D, Tsintzas K, Karpe F (2009) Circulating fibroblast growth factor 21 is induced by peroxisome proliferator-activated receptor agonists but not ketosis in man. J Clin Endocrinol Metab 94:3594-3601

19. Dushay J, Chui PC, Gopalakrishnan GS et al (2010) Increased fibroblast growth factor 21 in obesity and nonalcoholic fatty liver disease. Gastroenterology 139:456-463

20. Coskun T, Bina HA, Schneider MA et al (2008) Fibroblast growth factor 21 corrects obesity in mice. Endocrinology 149:6018-6027

21. Muller TD, Sullivan LM, Habegger K et al (2012) Restoration of leptin responsiveness in diet-induced obese mice using an optimized leptin analog in combination with exendin-4 or FGF21. J Pept Sci 18:383-393

22. Kharitonenkov A, Beals JM, Micanovic R et al (2013) Rational design of a fibroblast growth factor 21-based clinical candidate, LY2405319. PLoS One 8:e58575
23. Adams AC, Halstead CA, Hansen BC et al (2013) LY2405319, an engineered FGF21 variant, improves the metabolic status of diabetic monkeys. PLoS One 8:e65763

24. Gimeno RE, Moller DE (2014) FGF21-based pharmacotherapypotential utility for metabolic disorders. Trends Endocrinol Metab 25:303-311

25. Kharitonenkov A, Adams AC (2014) Inventing new medicines: the FGF21 story. Mol Metab 3:221-229

26. Bielohuby M, Menhofer D, Kirchner H et al (2011) Induction of ketosis in rats fed low-carbohydrate, high-fat diets depends on the relative abundance of dietary fat and protein. Am J Physiol Endocrinol Metab 300:E65-E76

27. Jornayvaz FR, Jurczak MJ, Lee HY et al (2010) A high-fat, ketogenic diet causes hepatic insulin resistance in mice, despite increasing energy expenditure and preventing weight gain. Am J Physiol Endocrinol Metab 299:E808-E815

28. Kennedy AR, Pissios P, Otu H et al (2007) A high-fat, ketogenic diet induces a unique metabolic state in mice. Am J Physiol Endocrinol Metab 292:E1724-E1739

29. Nishimura T, Nakatake Y, Konishi M, Itoh N (2000) Identification of a novel FGF, FGF-21, preferentially expressed in the liver. Biochim Biophys Acta 1492:203-206

30. Stemmer K, Kotzbeck P, Zani F et al (2015) Thermoneutral housing is a critical factor for immune function and diet-induced obesity in C57BL/6 nude mice. Int J Obes (Lond) 39:791-797

31. Wiese TJ, Lambeth DO, Ray PD (1991) The intracellular distribution and activities of phosphoenolpyruvate carboxykinase isozymes in various tissues of several mammals and birds. Comp Biochem Physiol B 100:297-302

32. Stark R, Pasquel F, Turcu A et al (2009) Phosphoenolpyruvate cycling via mitochondrial phosphoenolpyruvate carboxykinase links anaplerosis and mitochondrial GTP with insulin secretion. J Biol Chem 284:26578-26590

33. Stemmer K, Bielohuby M, Grayson BE et al (2013) Roux-en-Y gastric bypass surgery but not vertical sleeve gastrectomy decreases bone mass in male rats. Endocrinology 154:2015-2024

34. Pfaffl MW (2001) A new mathematical model for relative quantification in real-time RT-PCR. Nucleic Acids Res 29:e45

35. Lecker SH, Jagoe RT, Gilbert A et al (2004) Multiple types of skeletal muscle atrophy involve a common program of changes in gene expression. FASEB J 18:39-51

36. Yoon JC, Puigserver P, Chen G et al (2001) Control of hepatic gluconeogenesis through the transcriptional coactivator PGC-1. Nature 413:131-138

37. De Sousa-Coelho AL, Marrero PF, Haro D (2012) Activating transcription factor 4-dependent induction of FGF21 during amino acid deprivation. Biochem J 443:165-171

38. Pissios P, Hong S, Kennedy AR, Prasad D, Liu FF, Maratos-Flier E (2013) Methionine and choline regulate the metabolic phenotype of a ketogenic diet. Mol Metab 2:306-313

39. Laeger T, Henagan TM, Albarado DC et al (2014) FGF21 is an endocrine signal of protein restriction. J Clin Invest 124:3913-3922

40. Sarruf DA, Thaler JP, Morton GJ et al (2010) Fibroblast growth factor 21 action in the brain increases energy expenditure and insulin sensitivity in obese rats. Diabetes 59:1817-1824

41. Xu J, Lloyd DJ, Hale C et al (2009) Fibroblast growth factor 21 reverses hepatic steatosis, increases energy expenditure, and improves insulin sensitivity in diet-induced obese mice. Diabetes 58: 250-259

42. Owen BM, Ding X, Morgan DA et al (2014) FGF21 acts centrally to induce sympathetic nerve activity, energy expenditure, and weight loss. Cell Metab 20:670-677

43. Fisher FM, Estall JL, Adams AC et al (2011) Integrated regulation of hepatic metabolism by fibroblast growth factor 21 (FGF21) in vivo. Endocrinology 152:2996-3004 
44. Kharitonenkov A, Shiyanova TL, Koester A et al (2005) FGF-21 as a novel metabolic regulator. J Clin Invest 115: $1627-1635$

45. Kong LJ, Feng W, Wright $M$ et al (2013) FGF21 suppresses hepatic glucose production through the activation of atypical protein kinase Ciota/lambda. Eur J Pharmacol 702:302-308

46. Murata Y, Nishio K, Mochiyama T et al (2013) Fgf21 impairs adipocyte insulin sensitivity in mice fed a low-carbohydrate, highfat ketogenic diet. PLoS One 8:e69330 